\title{
Research Paper \\ Bioinformatic Prediction of miRNAs Targeting APRIL and BAFF Genes in Chronic Lymphocytic Leukemia
}

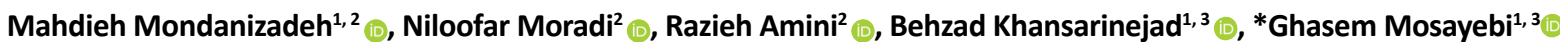

1. Molecular and Medical Research Center, Arak University of Medical Sciences, Arak University of Medical Sciences, Arak, Iran.

2. Department of Biotechnology and Molecular Medicine, School of Medicine, Arak University of Medical Sciences, Arak, Iran.

3. Department of Microbiology and Immunology, School of Paramedicine, Arak University of Medical Sciences, Arak, Iran.

\begin{tabular}{|l|l}
\hline $\begin{array}{l}\text { Use yourdevice to scan } \\
\text { and read the article online }\end{array}$ & $\begin{array}{l}\text { Citation: Mondanizadeh M, Moradi N, Amini R, Khansarinejad B, Mosayebi Gh. [Bioinformatic Prediction of miRNAs } \\
\text { Targeting APRIL and BAFF Genes in Chronic Lymphocytic Leukemia (Persian)]. Journal of Arak University of Medical } \\
\text { Sciences(JAMS). 2019; 22(5):32-43. https://doi.org/10.32598/JAMS.22.5.32 }\end{array}$ \\
doifhttps://doi.org/10.32598/JAMS.22.5.32
\end{tabular}

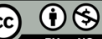

Article Info:

Received: 08 Jul 2019

Accepted: 27 Aug 2019

Available Online: 01 Dec 2019

Key words:

Bioinformatics

programs, Chronic

Lymphocytic Leu-

kemia, MicroRNA, APRIL, BAFF

\section{A B STRACT}

Background and Aim Chronic Lymphocytic Leukemia (CLL) is the most commonly occurring leukemia in adults, accounting for about $30-25 \%$ of total leukemia. One of the important etiological causes of this leukemia is the disruption of the Nuclear Factor Kappa B (NF-kB) signaling pathway. The two proteins of Apoptosis-Inducing Ligand (APRIL) and B-Cell Activating Factor (BAFF) play a role in the pathogenesis of this leukemia by affecting the NF-kB signaling pathway. In this study, due to the effect of miRNAs in regulating many cellular processes, the prediction of the prominent miRNAs targeting APRIL and BAFF transcripts in B-cell CLL patients was evaluated using specific and different bioinformatics programs.

Methods \& Materials Afterwards retrieving the sequences of APRIL and BAFF proteins from the NCBI website, by using several programs including miRanda, TargetScan, miRWalk, DIANA and miRDB with different algorithms, the prediction of miRNAs targeting these genes was investigated.

Ethical Considerations This study was approved by the Research Ethics Committee of Arak University of Medical Sciences.

Results Based on the scoring system of bioinformatics programs, "hsa-miR-145-5p" and "hsa-miR-185-5p" were identified as miRNAs targeting APRIL gene, while "hsa-miR-424" and "hsa-miR-497"were miRNAs targeting BAFF gene. They were suggested for the practical studies in future.

Conclusion Based on the important role of APRIL and BAFF genes in the normal process of cell death and B-cell evolution, it seems that the mi-RNAs predicted by bioinformatics programs using different algorithms can be used as a diagnostic molecular biomarker to identify B-cell CLL patients.

\section{Extended Abstract}

\section{Introduction}

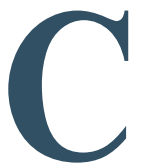

hronic Lymphocytic Leukemia (CLL) is the most common leukemia in adults that appears to be caused by a defect in cell differentiation. CLL has various etiologic causes includ- ing genetic alterations and impaired intracellular signaling pathway. Therefore, the identification and evaluation of proteins involved in molecular signaling pathway in the CLL that changes their expression relative to the normal state can lead to the development of a new and effective therapeutic or diagnostic method. The two proteins of Apoptosis-Inducing Ligand (APRIL) and B-Cell Activating Factor (BAFF) play

* Corresponding Author:

Ghasem Mosayebi, PhD

Address: Department of Microbiology and Immunology, School of Paramedicine, Arak University of Medical Sciences, Arak, Iran.

Tel: +98 (863) 4173503

E-mail: gmosayebi@yahoo.com 
a role in the pathogenesis of this leukemia by affecting the Nuclear Factor Kappa B (NF-kB) signaling pathway.

APRIL protein is significantly increased in the serum of patients with CLL, and by stimulating the NF- $\kappa \beta$ pathway, ultimately inhibits apoptosis in B-cells. On the other hand, BAFF protein, by helping B-cell lymphocyte proliferation, ultimately inhibits B-cell apoptosis and cell progression to tumorigenesis and become cancerous. In recent years, researchers have found that some miRNAs can cause cells to become cancerous by reducing gene expression and gene silencing. This confirms the important potential of these molecular agents as biomarkers in the diagnosis, prognosis, progression, treatment and drug resistance of cancers including CLL. In this regard, considering the effect of miRNAs in regulating many cellular processes, the prediction of the prominent miRNAs targeting APRIL and BAFF transcripts in B-cell CLL patients was evaluated in the present study using specific and different bioinformatics programs.

\section{Materials and Methods}

This study is based on the bioinformatics theory. First, the sequences of APRIL and BAFF proteins were retrieved from the NCBI website. Then, by using bioinformatics programs of miRanda, TargetScan, miRWalk, DIANA and miRDB, the prediction of miRNAs targeting these genes was investigated. These programs have different algorithms. The miRanda program presents the results of bioinformatics assessments by providing an index called "miRSVR", and the TargetScan program shows the results based on a factor called "Pct" (probability of conserved targeting) in humans and mammals, rats, worms, flies and frogs. Algorithm used in miRWalk software is based on prediction of miRNA binding site within complete sequences of all known genomes (even mitochondril genes).

The basis for prediction assessments in the DIANA program is an index called "miTG score" or precision score. In the miRDB database, the prediction score ranges from 50-100, and higher score shows the higher likelihood of miRNA binding to the target. Outputs from each program representing each miRNA score were then saved as an Excel file. Among the obtained data, miRNAs that the highest complementary to BAFF and APRIL genes were selected for the study.

\section{Results}

The results of the miRWalk program indicated that "miR15a-5p", "miR-500b-5p" and "miR-4803" were predicted and "miR-215" and "miR-192" were confirmed as BAFF gene targeters. The results of the DIANA program showed that the "miR-424" and "miR-497" targeted BAFF gene whose scores were 0.952 and 0.916 , respectively. The results of the miRDB program reported that "miR-15a-5p", "miR-497" and "miR-424" targeted BAFF gene with the highest scores. The results of the miRanda program showed that "hsa-miR-544" targets the BAFF gene.

The results of the TargetScan program indicated that the APRIL gene is targeted by "miR-145-5p" and "miR-5195" with the highest score and the best binding conditions. The results of the miRanda program showed that "miR-185" with the highest score and more efficient binding can target the APRIL gene. Based on the results of the DIANA program, APRIL gene is targeted by "miR-6132" and "miR185-5p" with the highest scores. According to the results of miRWalk program, numerous miRNAs both predicted and confirmed that were the targeters of APRIL gene. The results of miRDB database indicated that APRIL gene is targeted by hsa-miR-6716-5p and hsa-miR-4306 with the highest scores. Finally, "hsa-miR-145-5p" and "hsa-miR185-5p" were identified as miRNAs targeting APRIL gene, and "hsa-miR-424" and "hsa-miR-497" were miRNAs targeting BAFF gene. They were suggested for the practical studies in future.

\section{Conclusion}

Bioinformatics is an important technique for managing large-scale biological data. Previous studies have indicated that mi-RNAs are involved in almost all physiological and pathological mechanisms. Similarly, despite demonstrating the aberrant expression of miRNAs in many diseases including cancers, these molecular agents can be used as potential biomarkers for both diagnosis and treatment. Therefore, it seems that the prediction of the miRNAs that target BAFF and APRIL gene can be an effective step in the diagnosis and treatment of B-cell CLL.

To our knowledge, this is the first time that bioinformatics programs of miRanda, TargetScan, miRWalk, DIANA and miRDB are used for the target prediction of miRNAs. Based on the results, we found out that "hsa-miR-145-5p" and "hsa-miR-185-5p" were the miRNAs targeting APRIL gene, and "hsa-miR-424" and "hsa-miR-497" were targeting BAFF gene. Since there is no specific study on the role of these miRNAs in the progression of B-cell CLL, the present study, as the first study, allows the introduction and assessment of miRNAs and new genes to help diagnose patients with this cancer. 


\section{Ethical Considerations}

\section{Compliance with ethical guidelines}

This study obtained its ethical approval form the Research Ethics Committee of Arak University of Medical Sciences (Code: IR.ARAKMU.REC.1395.418).

\section{Funding}

This study extracted from a research proposal approved by the Arak University of Medical Sciences. We received financial support from the Deputy for Research and Technology of this University.

\section{Authors' contributions}

Conceptualization: Mahdieh Mondanizadeh and Ghasem Mosayebi; Investigation: Mahdieh Mondanizadeh, Niloofar Moradi and Razieh Amini; Review \& editing: Mahdieh Mondanizadeh and Niloofar Moradi; Validation: Behzad Khansarinejad; Supervision: Ghasem Mosayebi.

\section{Conflicts of interest}

The authors declare no conflict of interest.

\section{Acknowledgements}

The authors would like to thank the Deputy for Research and Technology and the staff of the Laboratory of Molecular Microbiology and Virology at Arak University of Medical Sciences and Pastor Institute for their support and cooperation. 


\title{
ييشبينى بيوانفورماتيكى BARNAهاى هدف تحيرنده ثرنهاى BAFF و APRIL در بيماران مبتلا به لوسمى لنفوسيتى مزمن بين
}

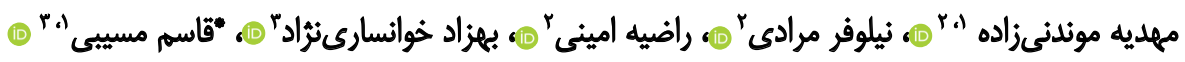 \\ 1. مركز تحقيقات بزُشكى و مولكولى، دانشكاه علوم يزشكى اراك، اراك، ايران.

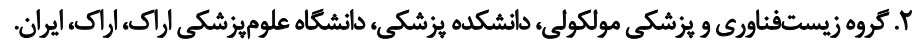

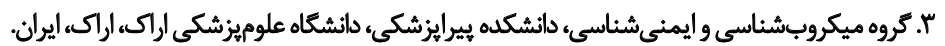

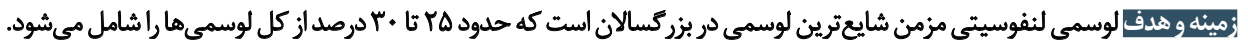

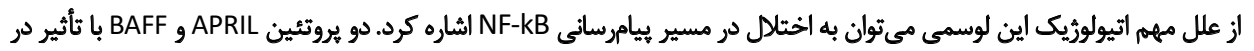

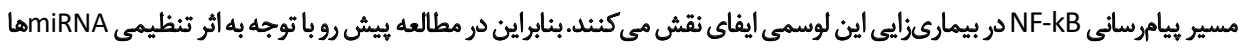

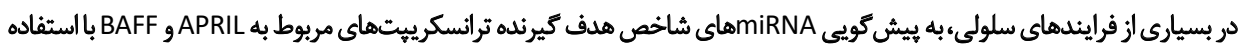

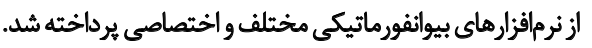

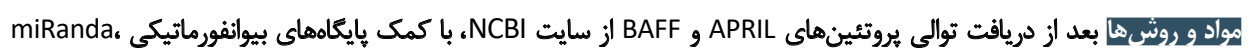

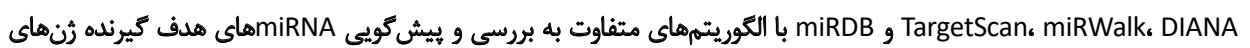

$$
\text { مذكور برداخته شد. }
$$

ملاحظات اخلاقيى اين مطالعه در كميته اخلاق دانشعاه علوم يزشكى اراى تصويب شده است.

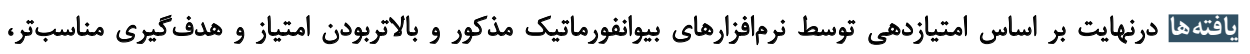

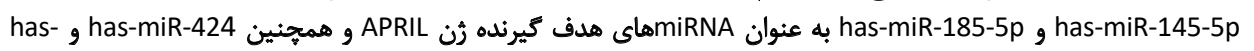

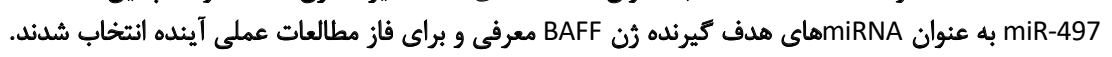

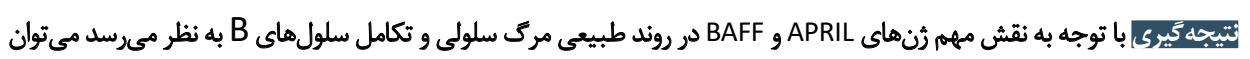

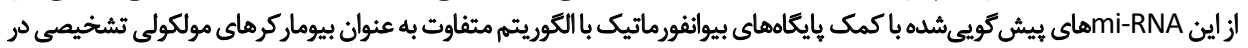

نظر آيد، قابليت مبارزه با عفونت را آنطور كه بايد داشته باشند،

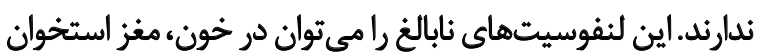

dates

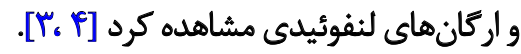
سرطان خون يكى از شايعترين سرطانهاست كه به نظر بهر لوسمى لنفوسيتى مزمن داراى سير بالينى متغيرى است؛

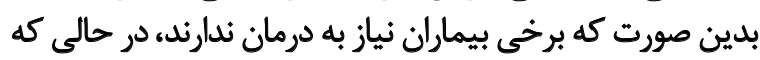

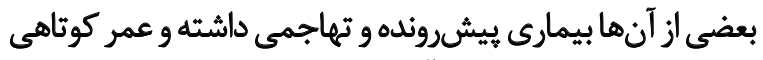

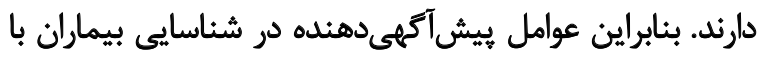

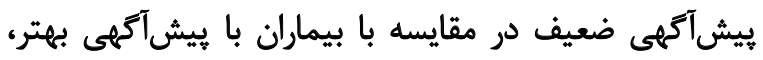

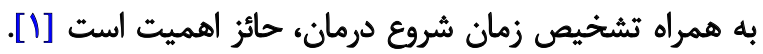

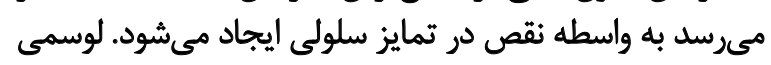

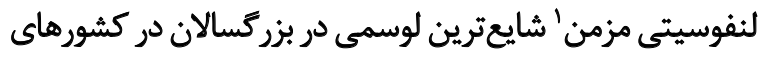

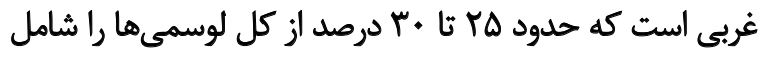

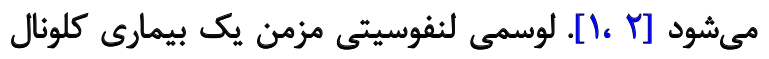

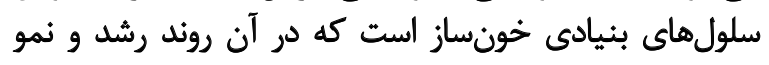

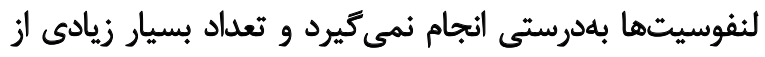

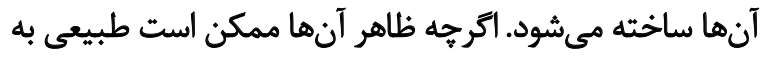

1. Chronic Lymphocytic Leukemia (CLL)

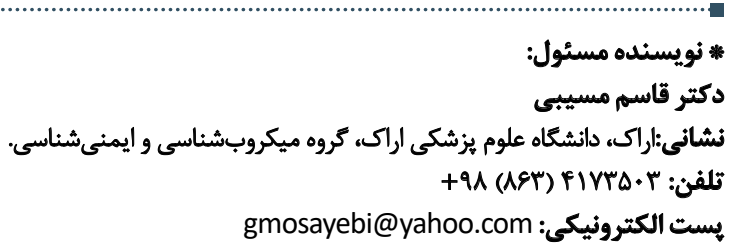


ها در بسيارى از فرايندهاى سلولى، به بيش

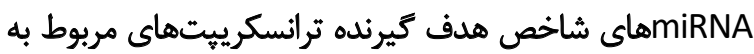
BAFF و APRIL

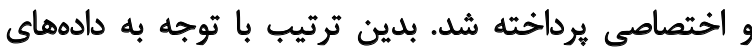

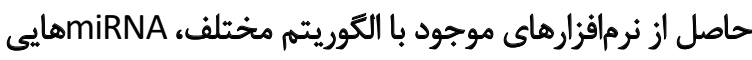

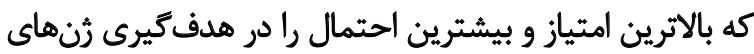

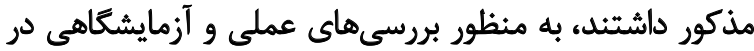
ئروهش هاى بعدى انتخاب شديند.

$$
\text { مواد و روشها }
$$

يُرؤشش حاضر يك بررسى تئورى بيواثفورماتيك است.

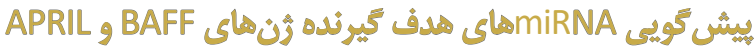

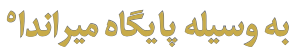

اين بايخاه اطلاعاتى \&نتايج حاصل از ارزيابى هائ بيوانفورماتيك

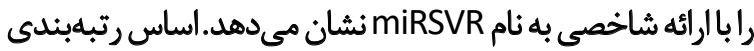

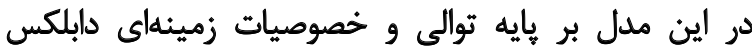

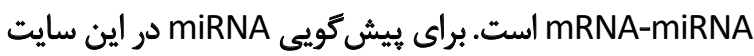
بعد از ورود به صفحه اصلى سايت وارد بخش

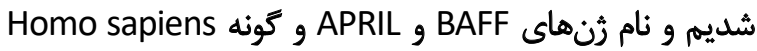

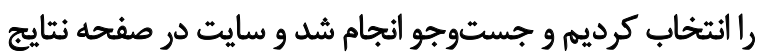

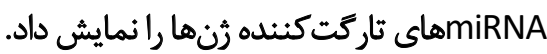

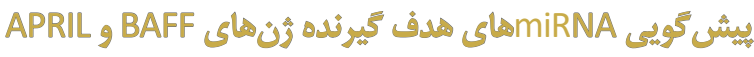

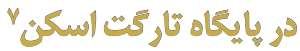

اين إيعاه اطلاعاتي ^نتايج حاصل از ارزيابى هاي بيوانفور ماتيك

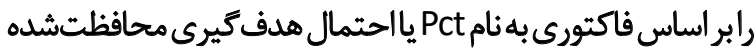

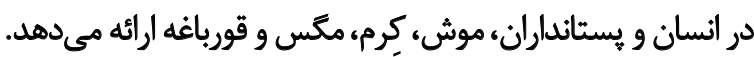

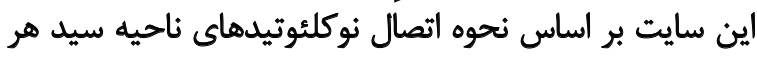

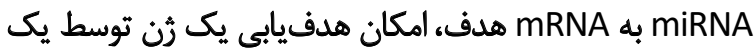

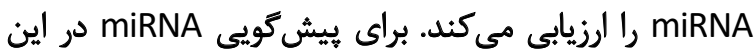

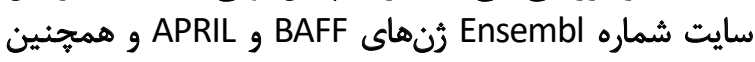

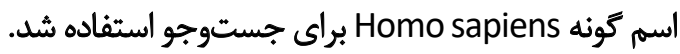

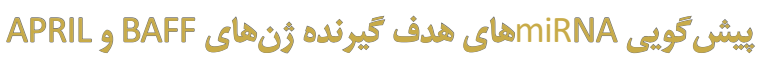

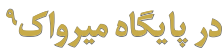

اين پايگاه داده"، اطلاعات هاي انسان، موش و رت

\section{MiRanda}

6. Http://www.microrna.org

7. TargetScan

8. Http://www.TargetScan

9. MiRWalk

10. Http://www.umm.uni-heidelberg.de/apps/zmf/mirwalk
بروز CLL به دو صورت تهاجمى ' و خفيف بمايان مىشود [ه].

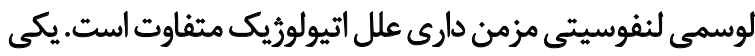

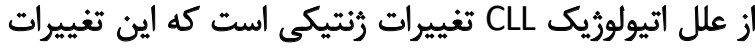

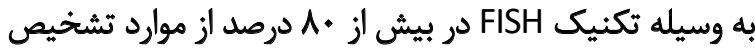

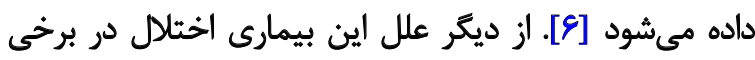

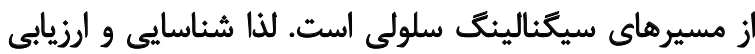

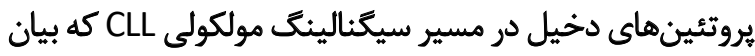

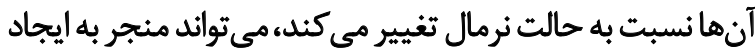

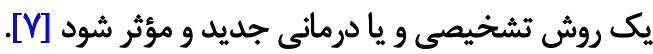

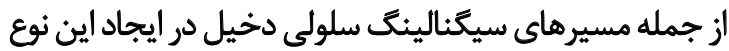

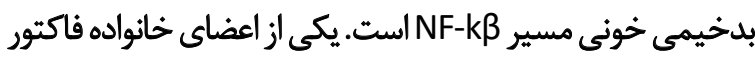

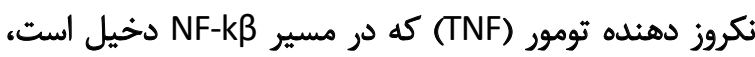

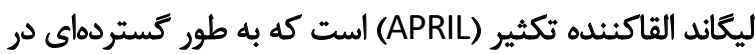

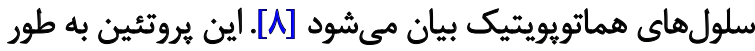

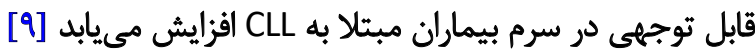

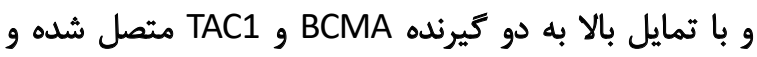

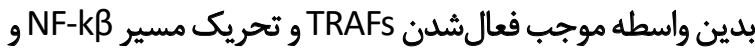

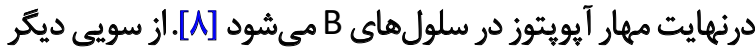

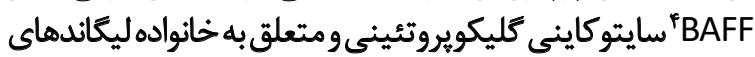

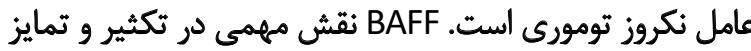

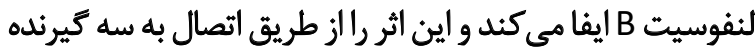

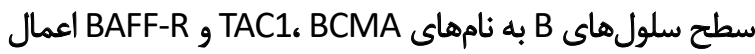

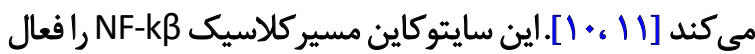
مي كند و با فعالشدن اين مسير BAFF همانند APRIL منجر

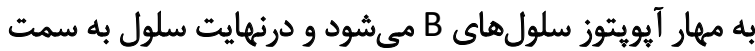

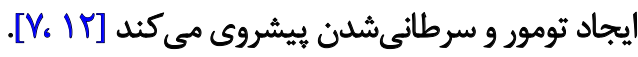

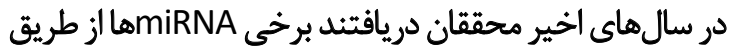

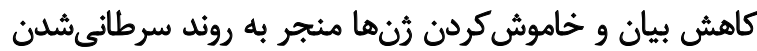

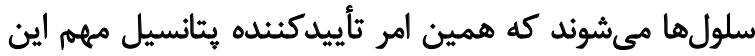

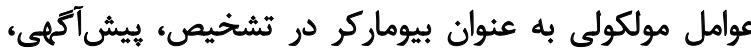

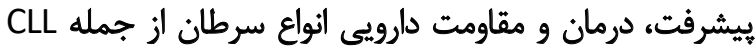

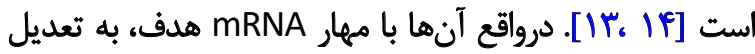

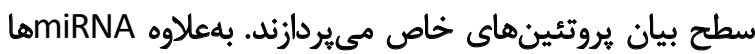

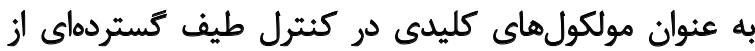

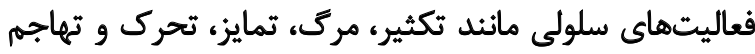

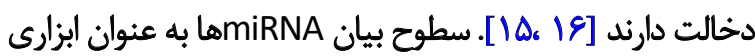

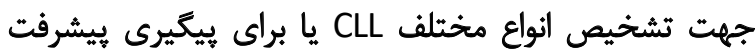

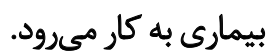

لذا در اين مطالعه با توجه به نقش كليدى يروتئينهاى

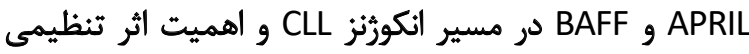

\section{Aggressive}

3. Indolent

4. B-cell Activating Factor (BAFF) 
يافتهها

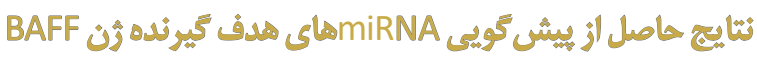

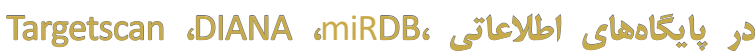
miRanda g miRWallk

miR195- نتان مي دهد Targetscan نتايج بررسى در بايكاه miR- و 5 ، ،miR15a-5p ،miR15b-5p ،miR-497 miR424

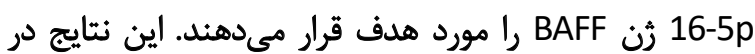
جدول شماره ا نشان داده شده است.

5تايج بررسى در بائاه miRWalk حاكى از اين بود كه miR-4803 miR-15a-5p ،miR-500b-5p

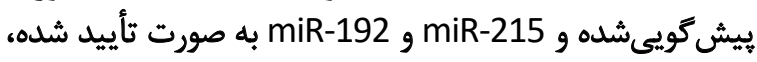

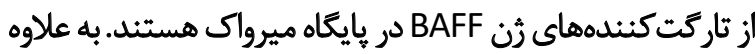

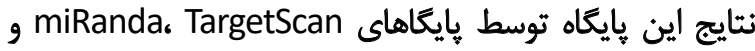

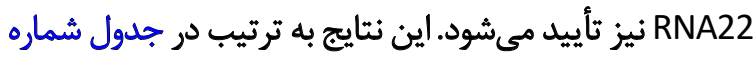

$$
\text { r بنشان داده شده است. }
$$

نتايج بررسى در غايكاه DIANA نشاندهنده هدفكيرى رن

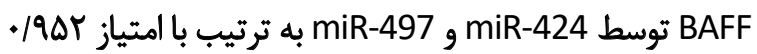

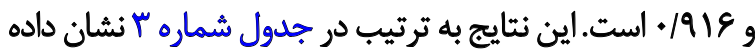

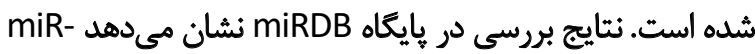

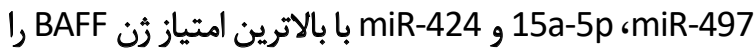
مورد هدف قرار مى دهند. نتايج در جدول شماره f قابل مشاهد باه

hsa- نشان مىدهد miRanda نتايج بررسى در يايخاه

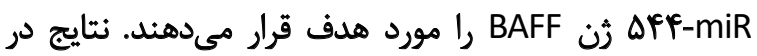
جدول شماره ه قابل مشاهده است.

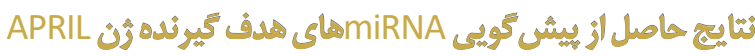

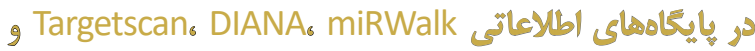 miRanda}

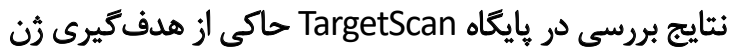

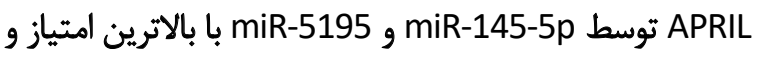

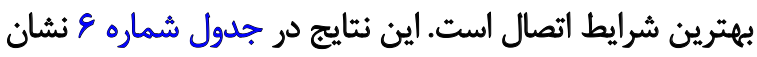

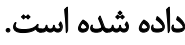

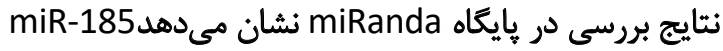

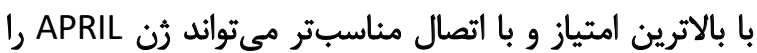

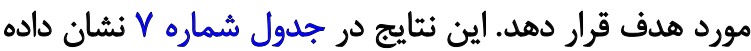

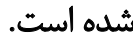

نتايج بررسى در بايعاه DIANA نشاندهنده هدفتيرى ثرن miR-185-5p g miR-6132 توساب بالاترين امتياز است.اين نتايج به ترثيب در جدول شماره منشان داده شده است.
را به دو صورت يُيشبينى شده و به صورت تجربى در اختيار

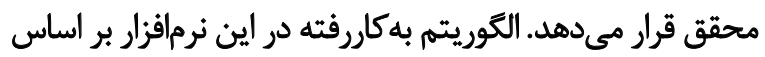

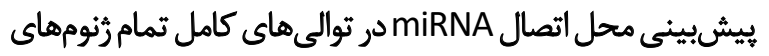

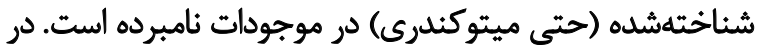

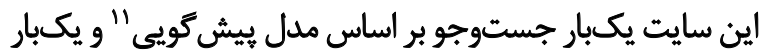

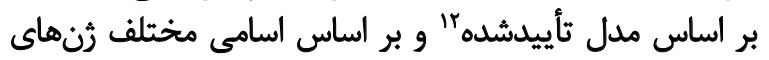
BAFF و APRIL انجام شد. تأبدشه.

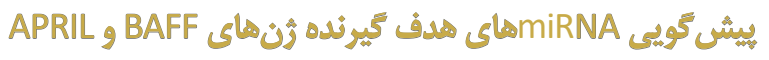

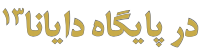

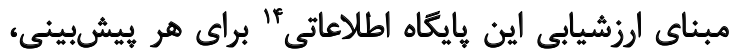

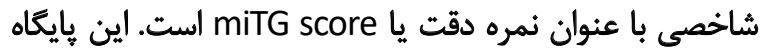

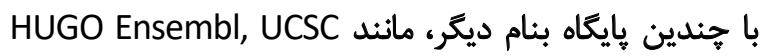
SwissProt

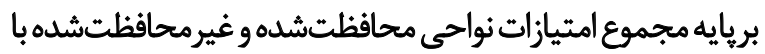

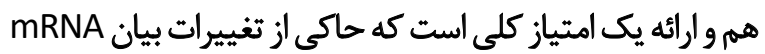

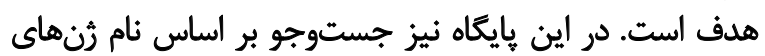

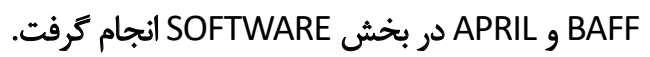

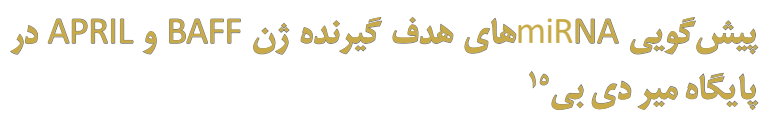
در اين بإيگاه اطلاعاتى آنلاين" "ِيشبينى miRNA براى بنج

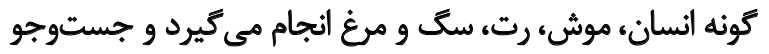

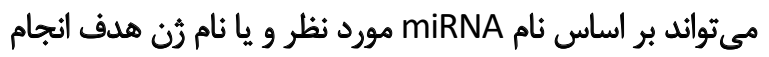

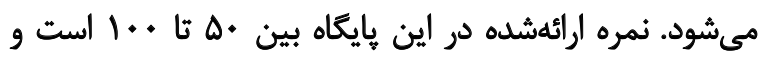

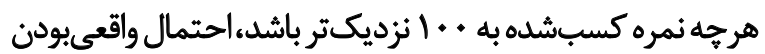

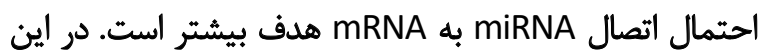

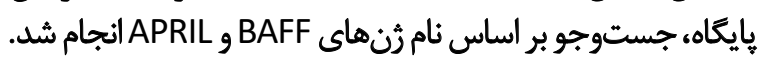

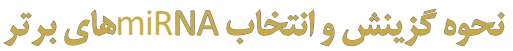

در ابتدا نتايج خروجى از هر بايخاه اطلاعاتى بر اساس

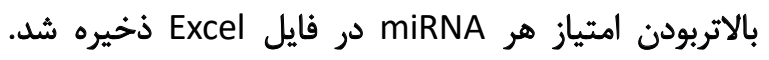

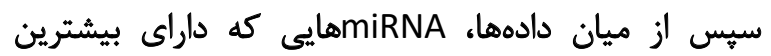

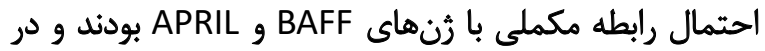

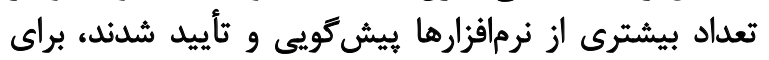
مطالعات عملى در آينده انتخاب شرمارئد
11. Predicted target module

12. Validated target module

13. DIANA

14. Http://diana.cslab.ece.ntua.gr/

15. $\mathrm{miRDB}$

16. Http://www.mirdb.org/ 


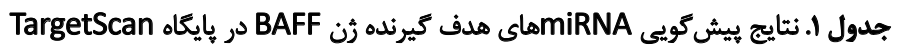

\begin{tabular}{|c|c|c|}
\hline Pct & نحوه اتصال • & miRNA \\
\hline.$/ \mathrm{Na}$ & 7 mer-m8 & has-miR-497 \\
\hline$+/ N 9$ & 7mer-m8 & has-miR-424 \\
\hline - IAV & 8 mer & has-miR-15b-5p \\
\hline.$/ 1$ & 8 mer & has-miR-195-5p \\
\hline.$/$ & $8 \mathrm{mer}$ & has-miR-16-5p \\
\hline
\end{tabular}

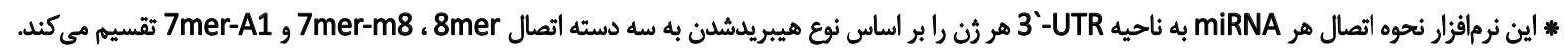

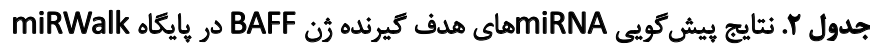

\begin{tabular}{cccccc}
\hline Targetscan & RNA22 & Miranda & miRWalk & miRNA Validated & miRNA Predicted \\
\hline$\checkmark$ & $\checkmark$ & $\checkmark$ & $\checkmark$ & has-miR-215 & has-miR-15a-5p \\
$\checkmark$ & $\checkmark$ & $\checkmark$ & $\checkmark$ & has-miR-192 & has-miR-500b-5p \\
\hline
\end{tabular}

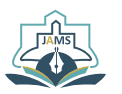

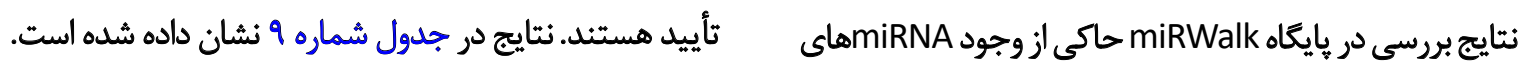
نثايج بررسى در بايعاه miRDB نشاندهئده هدف توسيرى ثن APRIL

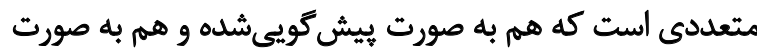

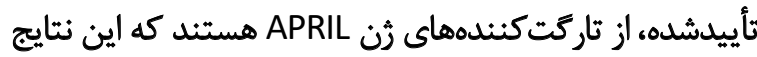
امتياز است. نتايج در جدول ثيه شماره · مأقابل مشاهده است.

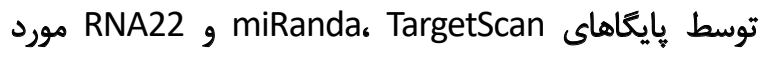

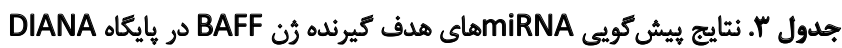

\begin{tabular}{cc}
\hline miTG score & miRNA \\
\hline / /ब४ & has-miR-424 \\
.$/ 919$ & has-miR-497 \\
.$/ 944$ & has-miR-195-5p \\
.$/ 194$ & has-miR-4739 \\
.$/ 99 \Delta$ & has-miR-16-5p \\
\hline
\end{tabular}

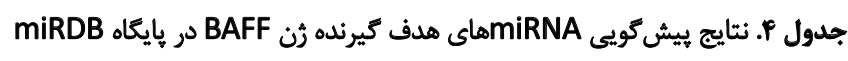

$\begin{array}{cc}\text { Target Score } & \text { miRNA } \\ \text { qr } & \text { hsa-miR-497 } \\ \text { qr } & \text { hsa-miR-424 } \\ \text { q. } & \text { hsa-miR-4803 } \\ \text { M } & \text { hsa-miR-500b-5p } \\ \text { \&V } & \text { hsa-miR-4739 }\end{array}$




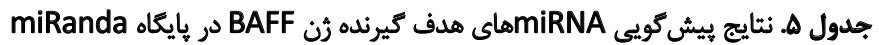

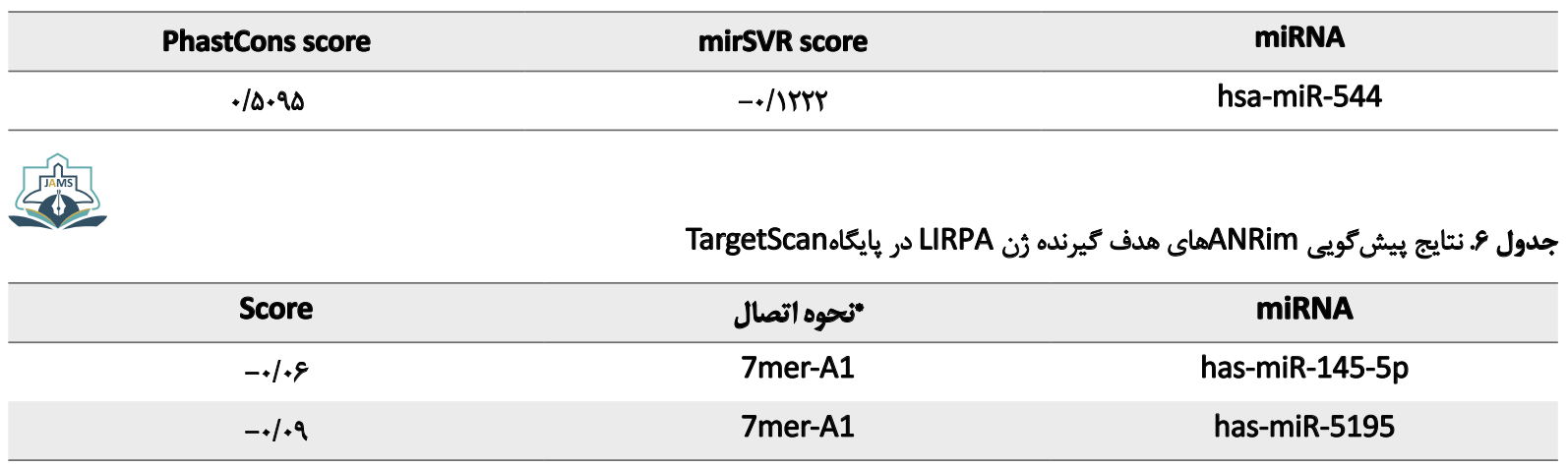

\section{tists}

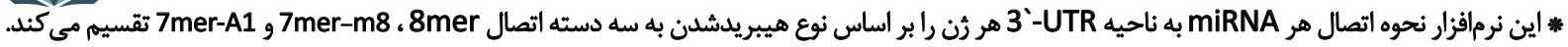

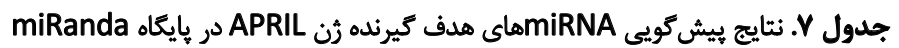

\begin{tabular}{|c|c|c|}
\hline PhastCons score & mirSVR score & miRNA \\
\hline$\cdot 18+\cdot v$ &.$- / 9401$ & hsa-miR-185-5p \\
\hline . $(\Delta 94)$ & $-1 / \cdot 1 f 8$ & hsa-miR-383 \\
\hline.$/ 8110$ & - - /fara & hsa-miR-222 \\
\hline . ISINA & - - /ffer & hsa-miR-221 \\
\hline
\end{tabular}

جدول A. نتايج بيش كويى هاى هدف كيرنده زن APRIL در بايكاه DIANA

\section{microT CDS}

. /9Ar

.$/ 911$

$.19 \cdot 9$

.$/ M$.

$+/ \mathrm{AYA}$

\section{MiRNA}

has-miR-6132

has-miR-185-5p

has-miR-4644

has-miR-4503

has-miR-1270

\section{$\sqrt[3]{3}$}

به سرطان خون و افراد كروه كنترل (افراد سالم) در مطالعات فاز

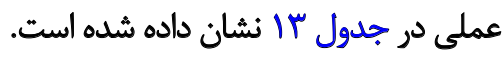

\section{بحث}

بيوانفورماتيك يك تكنيك مهرم براى مديريت دادهاى دري

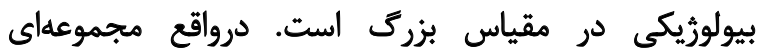

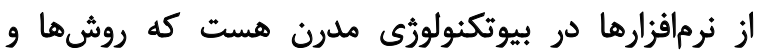

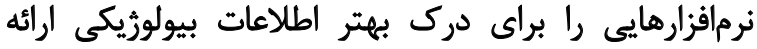

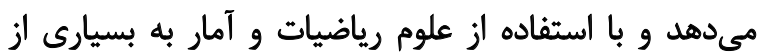

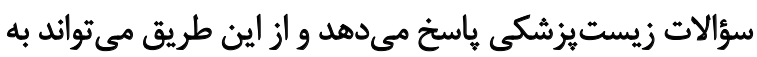

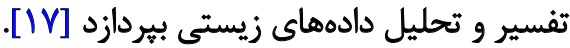

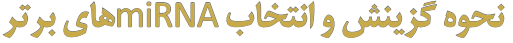
نحوه انتخاب و كزينش miRNA هدف كيرنده رن

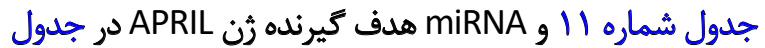

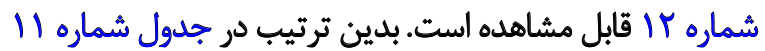

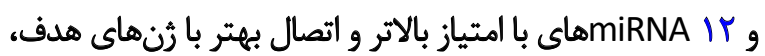

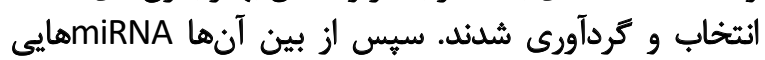

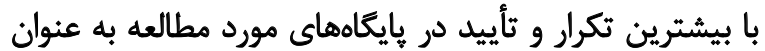

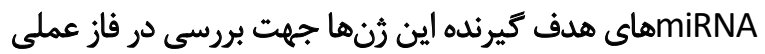

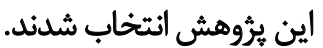
همجنين miRNA هاى منتخب miR-185، miR-145-5p (miR-497 ، براي بررسى ميزان بيان در افراد مبتلا (miR-424 


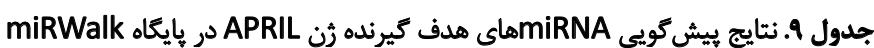

\begin{tabular}{|c|c|c|c|c|c|}
\hline Targetscan & RNA22 & miRanda & miRWalk & miRNA Validated & miRNA Predicted \\
\hline$\checkmark$ & $\checkmark$ & $\checkmark$ & $\checkmark$ & has-miR-548w & has-miR-1 \\
\hline$\checkmark$ & $\checkmark$ & $\checkmark$ & $\checkmark$ & has-miR-1293 & has-miR-330-3p \\
\hline$\checkmark$ & $\checkmark$ & $\checkmark$ & $\checkmark$ & has-miR-193b-3p & has-miR-3170 \\
\hline$\checkmark$ & $\checkmark$ & $\checkmark$ & $\checkmark$ & has-miR-320a & has-miR-206 \\
\hline$\checkmark$ & $\checkmark$ & $\checkmark$ & $\checkmark$ & has-miR-145-5p & has-miR-582-3p \\
\hline
\end{tabular}

جدول •ا. نتايج بيش تويي MiRNA هاى هدف كيرنده رن APRIL در بايكاه miRDB

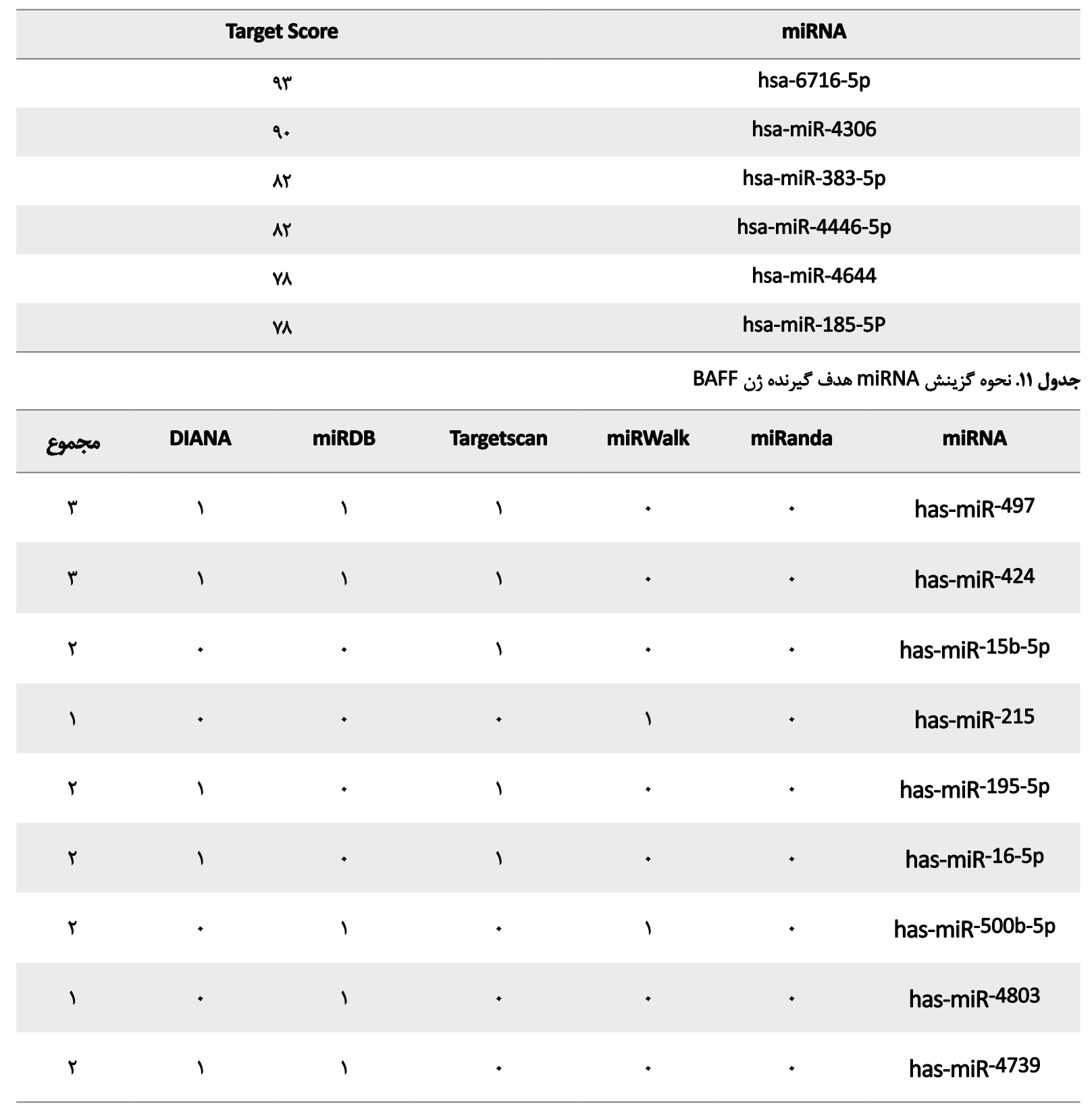




\begin{tabular}{|c|c|c|c|c|c|c|}
\hline \multirow[b]{2}{*}{ مجموع } & \multirow[b]{2}{*}{ miRanda } & \multirow[b]{2}{*}{ DIANA } & \multirow[b]{2}{*}{ Targetscan } & \multicolumn{3}{|c|}{ 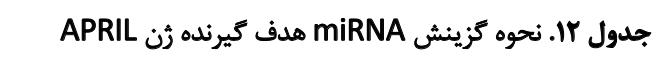 } \\
\hline & & & & miRWalk & miRDB & miRNA \\
\hline r & - & - & 1 & 1 & - & has-miR-145-5p \\
\hline r & 1 & 1 & . & • & 1 & has-miR-185-5p \\
\hline 1 & - & - & 1 & • & • & has-miR-5195 \\
\hline r & 1 & - & - & - & 1 & has-miR-383 \\
\hline 1 & 1 & - & - & - & - & has-miR-222 \\
\hline 1 & 1 & . & - & - & & has-miR-221 \\
\hline r & - & 1 & - & - & 1 & has-miR-6132 \\
\hline$r$ & • & 1 & - & + & 1 & has-miR-4644 \\
\hline 1 & • & 1 & • & $\cdot$ & + & has-miR-4503 \\
\hline 1 & - & 1 & - & $\cdot$ & . & has-miR-1270 \\
\hline 1 & - & . & - & 1 & - & has-miR-1 \\
\hline 1 & . & . & - & 1 & - & has-miR-330-3p \\
\hline 1 & . & . & . & 1 & . & has-miR-3170 \\
\hline 1 & . & • & . & 1 & . & has-miR-206 \\
\hline 1 & . & . & . & 1 & . & has-miR-582-3p \\
\hline
\end{tabular}

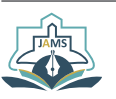

جدول ساى أ. أتخابشده جهت بررسى ميزان بيان در افراد مبتلا به لوسمى لنفوسيتى مزمن

\begin{tabular}{|c|c|c|}
\hline شماره شُناسايى & MiRNA & نام ثن \\
\hline \multirow[t]{2}{*}{ MI0001446 } & has-miR-424 & \\
\hline & & BAFF \\
\hline M10003138 & has-miR-497 & \\
\hline MI0000461 & has-miR-145-5p & \\
\hline & & APRIL \\
\hline MI0000482 & has-miR-185-5p & \\
\hline
\end{tabular}


به امتيازدهى در نرمافزارهاى بيوانفورماتيكى انتخاب و معرفى إنى

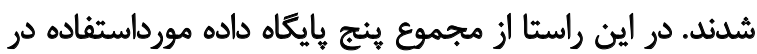

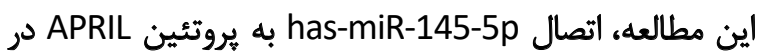

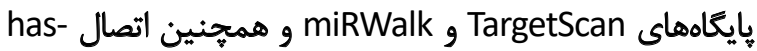

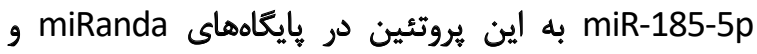

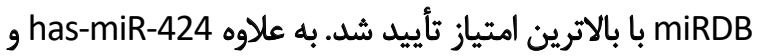

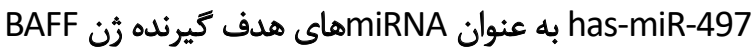

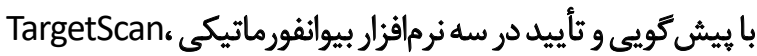

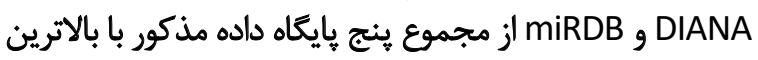

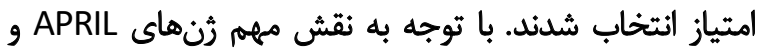

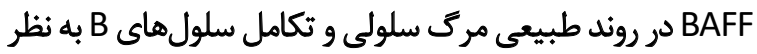

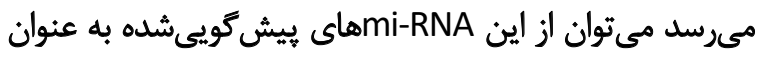
B- بيوماركرهاى مولكولى تشخيصى در جهت شناسايى بيماران CLL

\section{مالاحظات اخلاقى}

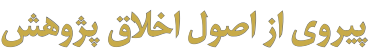

اين مطالعه در كميته اخلاق دانشكاه علوم يزشكى اراك با كد R.ARAKMU.REC.1395.418

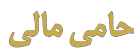

يروهش حاضر دربردارنده بخشى از طرح تحقيقاتى در دانشكاه

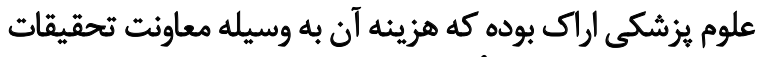

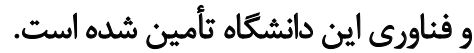

\section{هشار كت نُويسندأكان}

مفهومسازى: دكتر قاسم مسيبى و دكتر مهديه موندنىزادي؛ تحقيق و بررسى: نيلوفر مرادى، مرضيه

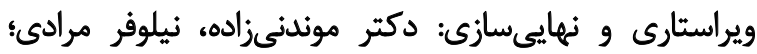

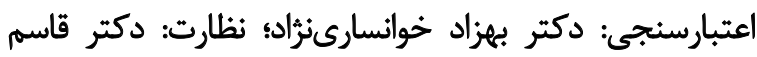

مسيبي

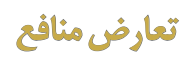

نويسند حان تصريح مى كنيد هيجزئه تضاد منافعى در خصوص ئروهش حاضر نداشتند.

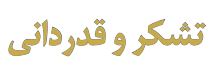

نويسندگان كمال قدرداني و امتنان را از اين معاونت دارند.

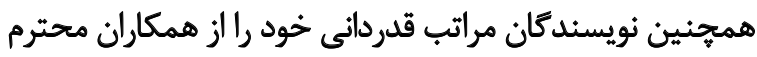

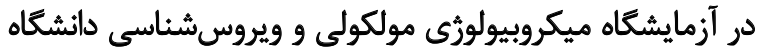
علوم يزشكى اراك و انستيتوياستور اعلام مي ودارند.
مطالعات بيشين حاكى از دخالت مها تقريباً در تمامى

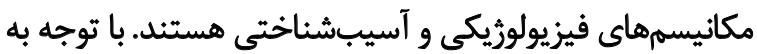

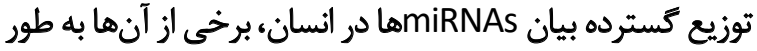

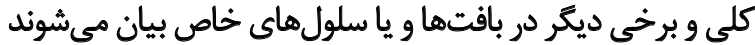

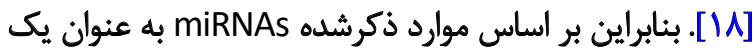

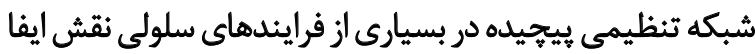

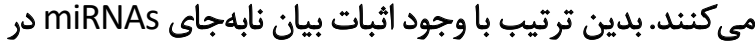

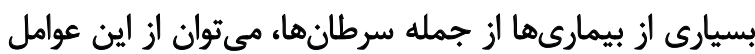

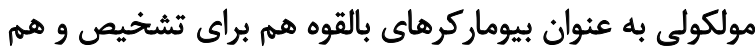

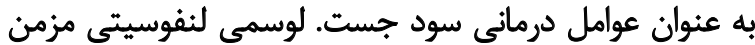

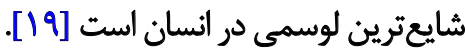

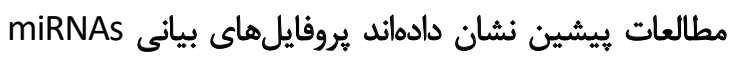

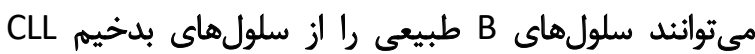

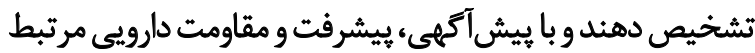

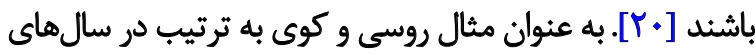

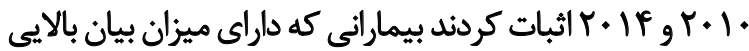

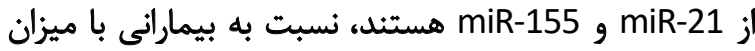

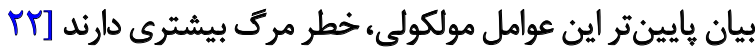

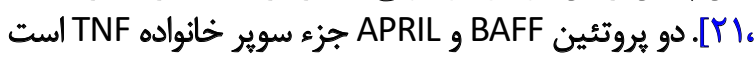

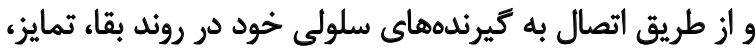

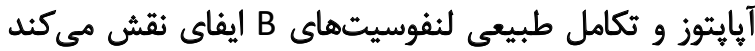

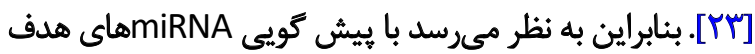

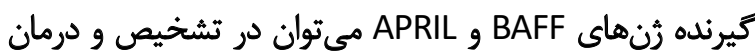

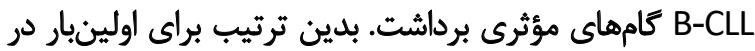

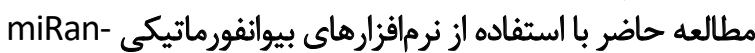
miRDB g da، TargetScan، miRWalk، DIANA

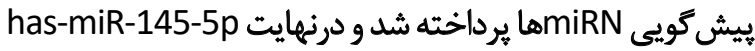

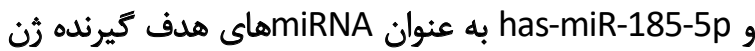

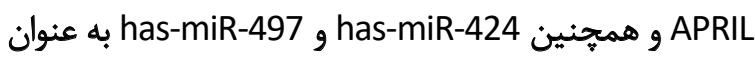
هاى هدف كيرنده رن BAFNA عملى آينده منتخب شدند.

از آنجايى كه براساس بررسى هاى انجامشده مطالعه اختصاصى

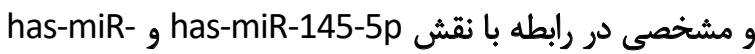

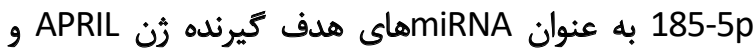

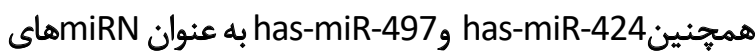
هدف كيرنده ثن BAFF در بيشرفت سرطان Ba

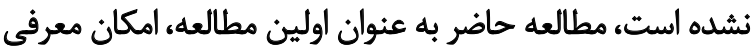

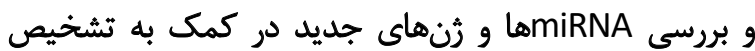
مبتلايان به اين سرطان را فراهم مي آورد.

$$
\text { تئيجهيرى }
$$

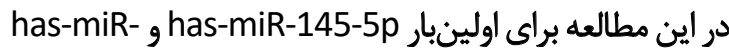
185-5p بر اساس احتمال اتصال مؤثرتر با زئ APRIL بارئ توجه 


\section{References}

[1] Ghia P, Stamatopoulos K, Belessi C, Moreno C, Stilgenbauer S, Stevenson $\mathrm{F}$, et al. ERIC recommendations on IGHV gene mutational status analysis in chronic lymphocytic leukemia. Leukemia. 2007; 21(1):1-3 [DOI:10.1038/sj.leu.2404457] [PMID]

[2] Gribben JG, O'Brien S. Update on therapy of chronic lymphocytic leukemia. J Clin Oncol. 2011; 29(5):544-50. [DOI:10.1200/JCO.2010.32.3865] [PMID] [PMCID]

[3] Jemal A, Siegel R, Xu J, Ward E. Cancer statistics, 2010. Cancer J Clin. 2010; 60(5):277-300. [DOI:10.3322/caac.20073] [PMID]

[4] Landgren O, Albitar M, Ma W, Abbasi F, Hayes RB, Ghia P, et al. B-cell clones as early markers for chronic lymphocytic leukemia. N Engl J Med. 2009; 360(7):659-67. [DOI:10.1056/NEJMoa0806122] [PMID]

[5] Alsagaby SA, Brennan P, Pepper C. Key molecular drivers of chronic lymphocytic leukemia. Clin Lymphoma Myeloma Leuk. 2016; 16(11):593606. [DOI:10.1016/j.clml.2016.08.008] [PMID]

[6] Döhner $H$, Stilgenbauer S, Benner A, Leupolt E, Kröber A, Bullinger $L$, et al. Genomic aberrations and survival in chronic lymphocytic leukemia. N Engl J Med. 2000; 343(26):1910-6. [DOI:10.1056/ NEJM200012283432602] [PMID]

[7] Boyd RS, Dyer MJ, Cain K. Proteomic analysis of B-cell malignancies. J proteomics. 2010; 73(10):1804-22. [DOI:10.1016/j.jprot.2010.03.010] [PMID]

[8] Zanesi N, Balatti V, Bottoni A, M Croce C, Pekarsky Y. Novel insights in molecular mechanisms of CLL. Curr Pharm Des. 2012; 18(23):3363-72. [DOI:10.2174/138161212801227104] [PMID]

[9] Planelles L, Carvalho-Pinto CE, Hardenberg G, Smaniotto S, Savino W, Gómez-Caro R, et al. APRIL promotes B-1 cell-associated neoplasm. Cancer Cell. 2004; 6(4):399-408. [DOI:10.1016/j.ccr.2004.08.033] [PMID]

[10] Shu HB, Hu WH, Johnson H. TALL-1 is a novel member of the TNF family that is down-regulated by mitogens. J Leukoc Biol. 1999; 65(5):680-3. [DOI:10.1002/jlb.65.5.680] [PMID]

[11] Kern C, Cornuel J-F, Billard C, Tang R, Rouillard D, Stenou V, et al. Involvement of BAFF and APRIL in the resistance to apoptosis of B-CLL through an autocrine pathway. Blood. 2004; 103(2):679-88. [DOI:10.1182/ blood-2003-02-0540] [PMID]

[12] Krappmann D, Vincendeau M, editors. Mechanisms of NF-KB deregulation in lymphoid malignancies. Semin Cancer Biol. 2016; 39:3-14. [DOI:10.1016/j.semcancer.2016.05.002] [PMID]

[13] Jansson MD, Lund AH. MicroRNA and cancer. Mol Oncol. 2012; 6(6):590-610. [DOI:10.1016/j.molonc.2012.09.006] [PMID] [PMCID]

[14] Mondanizadeh M, Mosayebi M, Arefian E, Saidijam M, Khansarinejad B. Bioinformatic evaluation of the miR-124 effect on transcription factors involved in neorogenesis process. Arak Med Univ J. 2014; 17(2):7381. [DOI:10.4264/numa.73.81]

[15] Lu YC, Chen YJ, Wang HM, Tsai CY, Chen WH, Huang YC, et al. Oncogenic function and early detection potential of miRNA-10b in oral cancer as identified by microRNA profiling. Cancer Prev Res (Phila). 2012; 5(4):665-74. [DOI:10.1158/1940-6207.CAPR-11-0358] [PMID]

[16] Moradi N, Paryan M, Khansarinejad B, Rafiei M, Mondanizadeh M. Bioinformatic prediction of miRNAs targeting Notch1 and HBx genes in chronic hepatitis B-induced hepatocellular carcinoma. Arak Med Univ J. 2017; 19(117):89-101.
[17] Akhtar MM, Micolucci L, Islam MS, Olivieri F, Procopio AD. Bioinformatic tools for microRNA dissection. Nucleic Acids Res. 2016; 44(1):24-44 [DOI:10.1093/nar/gkv1221] [PMID] [PMCID]

[18] Geng LY, Wang X. [Research progress of microRNA in chronic lymphocytic leukemia (Chinese)]. Zhongguo Shi Yan Xue Ye Xue Za Zhi. 2014; 22(1):255-8. [PMID]

[19] Balatti V, Acunzo M, Pekarky Y, Croce CM. Novel mechanisms of regulation of miRNAs in CLL. Trends Cancer. 2016; 2(3):134-43. [DOI:10.1016/j. trecan.2016.02.005] [PMID] [PMCID]

[20] Mirzaei H, Fathullahzadeh S, Khanmohammadi R, Darijani M, Momeni $\mathrm{F}$, Masoudifar A, et al. State of the art in microRNA as diagnostic and therapeutic biomarkers in chronic lymphocytic leukemia. J Cell Physiol. 2018; 233(2):888-900. [DOI:10.1002/jcp.25799] [PMID]

[21] Rossi S, Shimizu M, Barbarotto E, Nicoloso MS, Dimitri F, Sampath D, et al. microRNA fingerprinting of CLL patients with chromosome 17p deletion identify a miR-21 score that stratifies early survival. Blood. 2010 116(6):945-52. [DOI:10.1182/blood-2010-01-263889] [PMID] [PMCID]

[22] Cui B, Chen L, Zhang S, Mraz M, Fecteau JF, Yu J, et al. MicroRNA-155 influences B-cell receptor signaling and associates with aggressive disease in chronic lymphocytic leukemia. Blood. 2014; 124(4):546-54. [DOI:10.1182/blood-2014-03-559690] [PMID] [PMCID]

[23] Endo T, Nishio $M$, Enzler T, Cottam HB, Fukuda $T$, James DF, et al. BAFF and APRIL support chronic lymphocytic leukemia B-cell survival through activation of the canonical NF-kappaB pathway. Blood. 2007; 109(2):703-10. [DOI:10.1182/blood-2006-06-027755] [PMID] 